\title{
Caracterización de la salud visual y ocular en niños de un centro de desarrollo infantil de un barrio marginal de Pereira, Colombia
}

\author{
Characterization of visual and eye health in children of a development centre \\ from a marginal neighborhood in Pereira, Colombia
}

\author{
Mónica Marlene Márquez Galvis' ${ }^{1}$ Mayra Catalina Cáceres Díaz²
}

Para citar este artículo: Márquez MM, Cáceres MC. Caracterización de la salud visual y ocular en niños de un centro de desarrollo infantil de un barrio marginal de Pereira, Colombia. UstaSalud. 2017;16: 17-27.

\begin{abstract}
Licencia Creative Commons
\section{(c) (1) () $\Theta$} lo tanto, los lectores pueden acceder libremente a los artículos en su formato .pdf, igualmente podrán descargarlos y difundirlos; sin embargo no podrán modificarlos o alterarlos, adicionalmente se debe reconocer la autoría de las personas que figuran en las publicaciones, pero estas no podrán comercializadas.
\end{abstract}

\section{RESUMEN}

Objetivo: Describir las condiciones de la salud visual y ocular de los niños que asisten a un Centro de Desarrollo Infantil de un barrio marginal de la ciudad de Pereira, Colombia.

Materiales y métodos: Se describen las características sociodemográficas, visuales y oculares de niños que asisten a un Centro de Desarrollo Infantil (CDI) de la ciudad de Pereira, en edades comprendidas entre 9 a 70 meses, a partir de la información reportada en las historias clínicas de exámenes practicados entre los años 2012 y 2013.

Resultados: Se evaluaron 352 historias clínicas, 54,8\% correspondieron al género masculino. La edad media en la muestra fue de 49,7 meses. Los pacientes mestizos se presentaron en el $67,32 \%$ de los casos, siendo este grupo étnico el de mayor representación. La emetropía se observó en el $67,05 \%$, la hipermetropía en el $23,58 \%$, la miopía en un $9,38 \%$ y el astigmatismo se presentó en el $9,1 \%$ de los casos. El promedio de la esfera fue de +0.34 dioptrías, del astigmatismo 0.17 dioptrías y la mayoría de los pacientes presentaron ejes con la regla. De 704 ojos se halló $2,98 \%$ con blefaritis, en las etnias mestiza y afrocolombiana. La exoforia fisiológica está en el $42 \%$ de los casos.

Conclusiones: El estado refractivo más prevalente en todos los grupos de edad fue la emetropía, seguido por la hipermetropía. Las patologías de segmento anterior y anexos oculares fueron más frecuentes en las etnias mestiza y afrocolombiana, principalmente blefaritis y conjuntivitis. La exoforia y dentro de ella, la fisiológica, representa al estado motor más frecuentemente diagnosticado.

Palabras clave: Visión ocular, miopía, astigmatismo.

\section{ABSTRACT}

Objective: To describe the conditions of visual and ocular health of children attending a Child Development Center in a marginal neighborhood of the city of Pereira, Colombia.

Materials and methods: The sociodemographic, visual and ocular characteristics of children attending a Child Development Center (CDI) in the city of Pereira, between the ages of 9 and 70 months, are described from the information reported in the clinical records. Exam clinics practiced between 2012 and 2013.

Results: 352 clinical histories were evaluated, $54.8 \%$ corresponded to the masculine gender. The average age in the sample was 49.7 months. Mixed-race patients were presented in $67.32 \%$ of the cases, being this group the most common. Emmetropia was observed in $67.05 \%$, of the patients, hyperopia in $23.58 \%$, myopia in $9.38 \%$ and astigmatism occurred in $9.1 \%$ of cases. The average of the sphere was +0.34 Diopters, of astigmatism 0.17 Diopters and most of the patients presented axes with the rule. Of 704 eyes, $2.98 \%$ were found with blepharitis, in mixed and Afro-Colombian races. Physiological exophoria was in $42 \%$ of the cases.

Conclusions: The most prevalent refractive state in all age groups was emmetropia, followed by hyperopia. The pathologies of the anterior segment and ocular adnexa were more frequent in the mixed and Afro-Colombian ethnic groups, mainly blepharitis and conjunctivitis. The exophoria and within it, the physiological, represents the most frecuent motor state diagnosed in this study.

Keywords: Ocular vision, myopia, astigmatism.
${ }^{1}$ Grupo de Investigación Salud Visual Fundación Universitaria del Área Andina, Pereira, Colombia.

${ }^{2}$ Clínica OAT, Bogotá.

Autor de correspondencia:

Mónica Marlene Márquez Galvis

Correo electrónico:

mmmarquez@areandina.edu.co
Recibido para publicación
16 de mayo de 2017.
Aceptado para publicación:
15 de junio de 2017. 


\section{INTRODUCCIÓN}

El optómetra tiene una misión fundamental frente a la detección de las alteraciones en el desarrollo visual de los niños y la prevención de las complicaciones de los problemas visuales ${ }^{1}$; dichas alteraciones afectan el aprendizaje en la infancia, el desempeño en general de los individuos, e incluso en la edad adulta se han podido establecer asociaciones entre estas y dificultades en el acceso a diversos campos laborales, lo cual puede llevar incluso a un bajo nivel socioeconómico y en muchos casos a la exclusión social².

En Colombia, con la Resolución 4288 de 1996, la cual reglamenta el Plan de Atención Básica $(\mathrm{PAB})^{3}$ del Sistema General de Seguridad Social (SGSSS), se creó la guía para la detección oportuna de patologías oculares y alteraciones visuales; donde se indica la realización del tamizaje visual en los grupos de población de niños de 4 años y de 7 a 14 años, el examen optométrico en los casos detectados como positivos en el tamizaje y el examen oftalmológico en los recién nacidos prematuros. El Plan Decenal de Salud Pública 2012-2021 ${ }^{4}$, establece que tanto la salud visual, como la auditiva y la comunicativa, constituyen piezas indispensables para promover el desarrollo socioeconómico y luchar contra la pobreza.

La Organización Mundial de la Salud OMS ${ }^{5}$ estima que existen 19 millones de niños en condición de discapacidad visual en el mundo y 12 millones de esos casos son causados por defectos refractivos, fácilmente detectables y corregibles a través de los profesionales de la salud visual.

Morgan et al. ${ }^{6}$ en su estudio en ocho ciudades de diferentes países del mundo (incluida la ciudad de Santiago de Chile), y Figueroa y Molina ${ }^{7}$ en Colombia, reportaron prevalencias de hipermetropía en el grupo de 5 años de edad de más del $80 \%$. En otros trabajos, como el estudio MEPEDS ${ }^{8,9}$, se encontró una prevalencia del $20,8 \%, 12,7 \%$ y $6,6 \%$ para la hipermetropía, astigmatismo y miopía, respectivamente. Kleinstein et al. ${ }^{10}$ observaron que el $36,9 \%$ de los hispanos (mestizos) tiene astigmatismo, y en los afroamericanos un $6,4 \%$ presenta hipermetropía, $20 \%$ astigmatismo y 6,6\% miopía.

Medina ${ }^{11}$ en su estudio realizado en las diferentes comunidades indígenas colombianas reportó: "la mayoría de los pacientes examinados presentaron altera- ciones refractivas bajas, siendo la hipermetropía la de mayor prevalencia, en la mayoría de los casos se presentó en valores inferiores a $+1.50 \mathrm{D}$, esta se pudo asociar a defectos astigmáticos bajos contra la regla”.

En Irán un estudio evaluó 123 niños, en los cuales se halló que un $21,9 \%$ representaba discapacidad visual severa y ceguera ${ }^{12}$. Por otro lado, en India Dorairaj et al. ${ }^{13}$ observaron que de 13.241 niños, menos del $1 \%$ presentó ceguera causada por alteraciones del cristalino, anomalías del globo, distrofias retinales, glaucoma o atrofia óptica. En Nigeria, Rabiu ${ }^{14}$ en una población de 6.627 personas donde el $35,1 \%$ pertenecían al grupo de edad entre los 0 a 9 años, no observó baja visión.

En Perú, buscando establecer la asociación entre el rendimiento escolar y los trastornos de la visión, se observo que el $23 \%$ de los pacientes estudiados presentaba disminución de la agudeza visual ${ }^{15}$.

En Colombia, la encuesta del Departamento Administrativo Nacional de Estadística (DANE) ${ }^{16}$ en el censo poblacional de $2005^{16}$, reporta que de cada 1000 niños 8 presentan discapacidad visual, es decir, cerca de 18.952 menores de 5 años y 83.212 infantes que se encuentran entre los 5 y 11 años de edad.

Zuluaga et al. ${ }^{1}$ observaron que en Cali la discapacidad visual frecuentemente es producida por retinopatía de la prematuridad $(33,8 \%)$, presente en mayor medida en las niñas; seguida por el glaucoma y la toxoplasmosis. Por su parte, el censo realizado en el 2010 reportó para el departamento de Risaralda, 520 niños con discapacidad visual (baja visión y ceguera) entre 0 a 14 años ${ }^{17}$.

Por supuesto, es importante resaltar que los problemas no solamente están relacionados con la discapacidad visual y la ceguera, existen además, alteraciones como la ambliopía, cuya prevalencia se encuentra entre el $0,8 \%$ y $3,3 \%{ }^{18,19}$, siendo la causa más común de discapacidad visual en niños y adultos jóvenes ${ }^{20}$ y que de igual modo afecta varios aspectos a lo largo de la vida de las personas que la padecen ${ }^{21}$. Está alteración es causada principalmente por defectos refractivos y estrabismos no detectados tempranamente, cuyos efectos si se tratan oportunamente, pueden ser reversibles para la mayoría de los pacientes. 
El estrabismo es frecuente en la infancia, su prevalencia está estimada entre $1 \%$ y $3 \%$ en diferentes poblaciones ${ }^{22,23}$, está relacionado con una posición inadecuada de uno de los ojos, por lo que los ejes visuales de los dos ojos no están paralelos entre sí, evitando que sus fóveas sean estimuladas simultáneamente; la presentación temprana de esta alteración afecta el desarrollo de la binocularidad y la visión en general, causando ambliopía, entre otras alteraciones ${ }^{24}$.

Respecto a los defectos refractivos, en Nigeria, Rabiu $^{14}$ reportó en su estudio que solo el $0,2 \%$ de la población presentaba errores refractivos. Mientras que en Irán Ostadimoghaddam et al. ${ }^{25}$ reportaron una prevalencia del 5,2\% de miopía, 73,9\% de hipermetropía y $2 \%$ de astigmatismo en la población menor de 5 años, basándose en la refracción habitual (retinoscopía estática sin cicloplejia).

En Colombia, los estudios relacionados con el estado refractivo se encuentran principalmente reportados en municipios de los departamentos de Antioquia y Cundinamarca. En una población antioqueña, un estudio detectó una prevalencia de problemas visuales en menores de 12 años del 15,8\%, la hipermetropía se presentó en el 49,6\% de los casos (con exclusión de la hipermetropía fisiológica), seguido del 31,9\% por el astigmatismo; los problemas patológicos correspondieron al $0,83 \%$ y los ortopleópticos al $0,42 \%{ }^{26}$. Por su parte, Figueroa y Molina ${ }^{7}$ observaron en una población en Bogotá entre los 3 y los 12 años un 20\% de emetropía y $80 \%$ de hipermetropía.

En su estudio realizado en el litoral del Chocó, Buchelli y Corva en el $2009^{27}$, reportaron la presencia de la conjuntivitis bacteriana en aproximadamente 200 casos por cada 1.000 individuos en la población indígena Wounana y conjuntivitis alérgica en 20 de cada 1.000 individuos del género femenino en población afrocolombiana.

La refracción en los niños es usualmente hipermetrópica, y va cambiando progresivamente a la emetropía a lo largo de los primeros años. Según los datos presentados por Atkinson et al. ${ }^{28}$ en un grupo de pacientes pediátricos se observó una disminución representativa de la hipermetropía, durante el segundo y tercer año de vida, así como en la esfera y el cilindro. A esta reducción en el estado refractivo que ocurre durante los primeros años de vida se le denomina eme- tropización. Sin embargo, este mecanismo es sensible de alterarse por diversas causas, entre ellas las heredofamiliares, la prematuridad, las patologías oculares, los hábitos, el ambiente, la experiencia visual y la influencia nutricional ${ }^{29}$ afectan el estado refractivo, el crecimiento ocular y, por consiguiente, el desarrollo visual.

En Risaralda y específicamente en Pereira no existen datos de la prevalencia de alteraciones visuales en niños, por lo cual es necesario realizar estudios que caractericen a las poblaciones en esta edad vulnerable.

El presente artículo describe el estado de la salud visual y ocular de los niños que asisten a un Centro de Desarrollo Infantil (CDI) de la ciudad de Pereira, donde predominan los estratos socioeconómicos 0 y 1 .

Por lo antes expuesto, se planteó en este estudio describir el estado de la salud visual y ocular de niños de un Centro de Desarrollo Infantil (CDI) de la ciudad de Pereira.

\section{MATERIALES Y MÉTODOS}

El Programa de Optometría del Área Andina Seccional Pereira, como miembro del grupo Sociedad en Movimiento, "Programa de una sociedad y economía basada en el conocimiento", evaluó la salud visual y ocular de los niños que asisten al Centro de Desarrollo Infantil (CDI) Perlitas del Otún, durante los años 2012 y 2013, esta población proviene de familias que se encuentran en condición de desplazamiento y minorías étnicas (afrocolombianos ${ }^{30,31}$ y Embera Chamín ${ }^{32,33}$ ). El CDI es una modalidad de educación inicial,

...instituciones dirigidas a atender y promover un desarrollo integral a través de la educación inicial, con la participación de profesionales idóneos en temas relacionados con los diferentes componentes de la atención integral, responsables de gestionar las condiciones materiales que hacen efectivos todos los derechos de los niños y niñas en primera infancia..." ${ }^{34}$.

El Ministerio de Educación reconoce que en las diferentes zonas del país requieren de un funcionamiento especial para la atención de la primera infancia con grupos étnicos, teniendo en cuenta las particularidades regionales ${ }^{35}$. 
Se realizó un estudio de corte transversal ${ }^{36}$ que describe las características sociodemográficas, la morbilidad visual y ocular (determinando la frecuencia de patologías del segmento anterior, anexos oculares, estados motores y refractivos). Mediante notación vectorial se determinó el promedio del estado refractivo del ojo derecho hallado con retinoscopía estática sin cicloplejia de los niños que asistian a un CDI de Pereira, a partir de los datos consignados en las historias clínicas de valoraciones entre el 2012 y 2013.

La recolección de la información fue obtenida de las historias clínicas; y fue compilada en una base de datos donde se agrupó para obtener la información de edad, género, etnia, estado motor, estado refractivo (retinoscopía sin cicloplejia) y disposición. Se aplicó estadística descriptiva; tablas de frecuencias y porcentajes utilizando el programa SPSS 17.0 (SPSS Inc. Released 2008. SPSS Statistics for Windows, Version 17.0. Chicago: SPSS Inc.), se hizo análisis univariado de la edad, etnia y género (descrito por Márquez y Cáceres en el proyecto macro ${ }^{33}$ ), puntaje del Sistema de Selección de Beneficiarios para Programas Sociales (SISBÉN) el cual, a través de un puntaje, clasifica a la población según sus condiciones socioeconómicas ${ }^{37}$, estado motor y patológico, prescripciones ópticas, farmacológicas y remisiones; de igual manera, se realizó un análisis bivariado para el grupo de edad con estado refractivo, etnia con estado refractivo y grupo de edad versus estado motor, etnia con estado refractivo y grupo de edad con estado motor.

Con la identificación registrada en las historias clínicas se consultó en la página en Internet del Fondo de Seguridad y Garantía (FOSYGA) para determinar el tipo de afiliación en salud de cada niño, la cual puede pertenecer al régimen contributivo o al Plan Obligatorio de Salud POS (a este régimen "se deben afiliar las personas que tienen una vinculación laboral, es decir, con capacidad de pago como los trabajadores formales e independientes, los pensionados y sus familias"38) o Plan Obligatorio de Salud-subsidiado POS-S ("en el Régimen Subsidiado en Salud deben estar afiliadas las personas de escasos recursos, vulnerables del país, es decir, las clasificadas en los niveles I o II del Sisbén, y las poblaciones prioritarias especiales..." ${ }^{39}$ ).

En la página del Sisbén se consultó el puntaje de clasificación de los pacientes; el cual se calcula en este sistema con base en los datos suministrados en la encuesta, la puntuación va de cero (0) a (100), el puntaje obtenido permite identificar la población en condiciones de vulnerabilidad ${ }^{37}$.

La definición de los estados refractivos se realizó de la siguiente manera: Emetropía entre $(-0,25$ y $+0,50)$, Hipermetropía $(\geq+0,75)$, Miopía $(\geq-0,50)$, Astigmatismo ( $\geq-0,75)$, Anisometropía (diferencia en esfera y/o cilindro $\geq 1,00$ ). Los valores refractivos fueron analizados a través de los métodos de equivalente esférico y vectorial.

La investigación sobre registros de historias clínicas no involucra intervenciones ni modificaciones de variables biológicas, fisiológicas, psicológicas o sociales. Los datos fueron tratados de manera global sin identificar casos particulares. De acuerdo con la Resolución 008430 del 4 de octubre de 1993, artículo $11^{40}$ del Ministerio de la Protección Social, esta es una investigación que no involucra riesgo.

\section{RESULTADOS}

Se evaluaron 352 historias clínicas, encontrándose que $193(54,8 \%)$ pertenecen al género masculino. La edad media correspondió a 49,7 \pm 12 meses, las edades mínima y máxima corresponden a 11,7 meses y 69,4 meses, respectivamente. La mayor frecuencia se presentó en el grupo de los 49 a 60 meses de edad con 150 historias clínicas $(42,6 \%)$.

La representación por grupos étnicos fue para el mestizo el 67,3\% (237), seguido por el afrocolombiano $27,8 \%$ (98) y el indígena el 4,9\% (17) (figura 1).

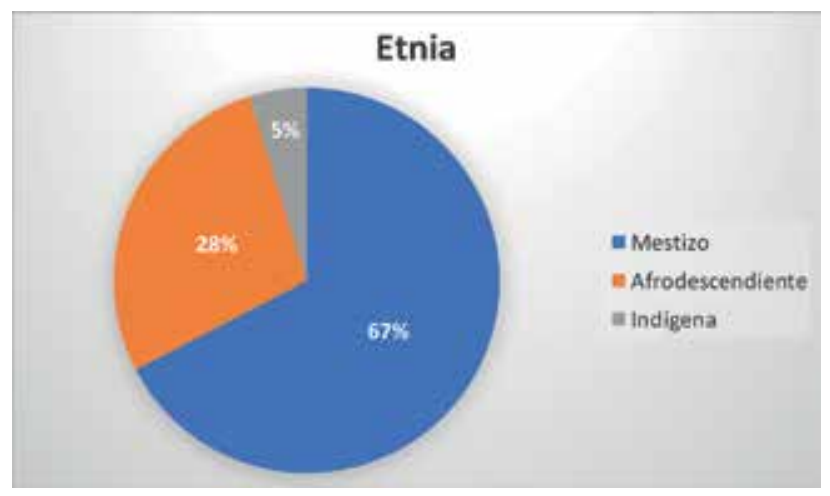

Figura 1. Representación por grupo étnico. 
El 62,2\% (219) se encontraba afiliado en el POS-S, el $32,7 \%$ (115) en el POS y el 5,1\% (18) sin afiliación en FOSYGA (figura 2). El promedio del puntaje obtenido en el SISBÉN correspondió a 29,0 $\pm 15,4$ (mínimo 3,1 y máximo 67,9); el 21,3\% (75) no se encontraban registrados en Sisbén.

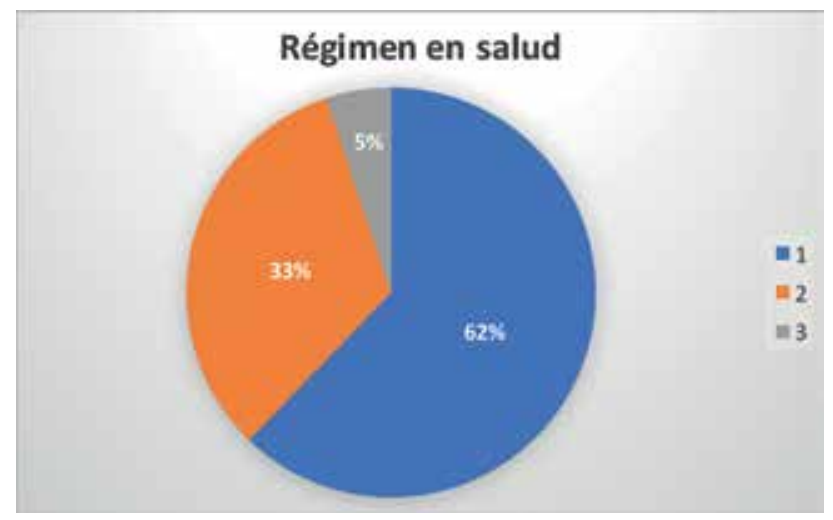

Figura 2. Tipo de afiliación al régimen de salud.

Al dato refractivo se le aplicó el análisis del equivalente esférico, con un promedio de $+0,25 \pm 1,18$ Dioptrías (D); la comparación con el método vectorial muestra una diferencia que clínicamente no es significativa (0,09 D). Teniendo en cuenta que el análisis mediante el método vectorial discrimina eje, esfera y cilindro, este fue el elegido para este estudio.

La emetropía se observó en el 69\% de la muestra (243), la hipermetropía en el 19\% (67), el astigmatis- mo estuvo representado por el 9,1\% (32), mientras que la miopía se presentó solo en el 2,8\% (10), los astigmatismos hallados se encontraron combinados con hipermetropía principalmente. El equivalente esférico promedio para la población correspondió a $+0,34 \pm 1,46 \mathrm{D}$ y el cilindro $0,17 \pm 0,57 \mathrm{D}$ (J0) con una tendencia de presentación de ejes con la regla (J45) $\left(179,5^{\circ} \mathrm{DS} \pm 8,6\right)$, Tabla 1 . La anisometropía fue hallada en el 6,8\% de las historias clínicas al comparar los estados refractivos de ambos ojos.

Como se observa en la Tabla 2 (zona gris oscura), el estado refractivo en la esfera con mayor porcentaje en los tres grupos étnicos correspondió a la emetropía, seguido por la hipermetropía baja con el 29,4\% (6) en los indígenas, $13,3 \%$ (20) en los afrocolombianos y $8,9 \%$ (35) en los mestizos (zona gris pálida superior), en total el 27,6\% (28) de los afrocolombianos, el 47,1\% (8) de los indígenas y el 19,8\% (47) de los mestizos son hipermétropes, La miopía baja se presentó en los grupos afrocolombiano y mestizo (zona gris pálida inferior).

En el total de la población mestiza la hipermetropía se presentó en el 19,8\% (47), la miopía en el 2,5\% (6), el astigmatismo en el 8,4\% (20). El 2,6\% de la población (9) presentó estados refractivos no fisiológicos (hipermetropía mayor a $2.5 \mathrm{D}$ o miopía mayor a $1.00 \mathrm{D}$ ). Los estados refractivos altos o extremos se presentaron solo en el grupo mestizo.

Tabla 1. Frecuencia de valores refractivos por grupos de edad

\begin{tabular}{ccccccccc}
\hline Grupos de edad (meses) & Emetropía & Porcentaje & Miopía & Porcentaje & Hipermetropía & Porcentaje & Astigmatismo & Porcentaje \\
\hline $12-18$ & 2 & 0,6 & 0 & 0,0 & 0 & 0,0 & 1 & 0,3 \\
$19-24$ & 4 & 1,1 & 2 & 0,6 & 3 & 0,9 & 0 & 0,0 \\
$25-30$ & 6 & 1,7 & 0 & 0,0 & 2 & 0,6 & 2 & 2 \\
$31-36$ & 26 & 7,4 & 1 & 0,3 & 7 & 2,0 & 0,6 & 0,6 \\
$37-42$ & 38 & 10,8 & 0 & 0,0 & 4 & 1,1 & 6 & 1,7 \\
$43-48$ & 21 & 6,0 & 2 & 0,6 & 4 & 1,1 & 4 & 1,1 \\
$49-60$ & 106 & 30,1 & 3 & 0,9 & 30 & 8,5 & 11 & 3,1 \\
$61-72$ & 40 & 11,4 & 2 & 0,6 & 17 & 4,8 & 6 & 1,7 \\
\hline Total & 243 & 69,0 & 10 & 2,8 & 67 & 19,0 & 32 \\
\hline
\end{tabular}


Tabla 2. Frecuencia de la emetropía y ametropía esférica por grupos étnicos

\begin{tabular}{|c|c|c|c|c|c|c|}
\hline \multirow[b]{2}{*}{ Esfera } & \multicolumn{2}{|c|}{ Afrocolombiano } & \multicolumn{2}{|c|}{ Indígena } & \multicolumn{2}{|c|}{ Mestizo } \\
\hline & Frecuencia & Porcentaje & Frecuencia & Porcentaje & Frecuencia & Porcentaje \\
\hline 8,75 & 0 & 0,0 & 0 & 0,0 & 1 & 0,4 \\
\hline 7 & 0 & 0,0 & 0 & 0,0 & 1 & 0,4 \\
\hline 3,75 & 1 & 1,0 & 0 & 0,0 & 1 & 0,4 \\
\hline 3,25 & 1 & 1,0 & 0 & 0,0 & 0 & 0,0 \\
\hline 2,75 & 1 & 1,0 & 0 & 0,0 & 0 & 0,0 \\
\hline 2,5 & 0 & 0,0 & 0 & 0,0 & 3 & 1,3 \\
\hline 2 & 2 & 2,0 & 1 & 5,8 & 1 & 0,4 \\
\hline 1,75 & 2 & 2,0 & 1 & 5,9 & 1 & 0,4 \\
\hline 1,5 & 1 & 1,0 & 0 & 0,0 & 4 & 1,7 \\
\hline 1,25 & 2 & 2,0 & 1 & 5,9 & 7 & 3,0 \\
\hline 1 & 5 & 5,1 & 0 & 0,0 & 7 & 3,0 \\
\hline 0,75 & 13 & 13,3 & 5 & 29,4 & 21 & 8,9 \\
\hline 0,5 & 16 & 16,3 & 2 & 11,8 & 56 & 23,6 \\
\hline 0,25 & 23 & 23,5 & 2 & 11,8 & 55 & 23,2 \\
\hline 0 & 19 & 19,4 & 5 & 29,4 & 59 & 24,9 \\
\hline$-0,25$ & 8 & 8,4 & 0 & 0,0 & 14 & 5,9 \\
\hline$-0,5$ & 2 & 2,0 & 0 & 0,0 & 4 & 1,7 \\
\hline$-0,75$ & 0 & 0,0 & 0 & 0,0 & 1 & 0,4 \\
\hline$-1,25$ & 1 & 1,0 & 0 & 0,0 & 0 & 0,0 \\
\hline$-1,5$ & 1 & 1,0 & 0 & 0,0 & 0 & 0,0 \\
\hline-15 & 0 & 0,0 & 0 & 0,0 & 1 & 0,4 \\
\hline Totales & 98 & 100,0 & 17 & 100,0 & 237 & 100,0 \\
\hline
\end{tabular}

La tabla 3 representa el astigmatismo bajo (zona gris oscura, que corresponde a los individuos que de acuerdo con el criterio del estudio se consideraron esféricos), se encontró en los grupos étnicos con una frecuencia del $33,3 \%$ (3), 11,2\% (11) y 10,9\% (5) para el indígena, mestizo y afrocolombiano, respectivamente. El astigmatismo alto se observó en la etnia mestiza seguido por la afrocolombiana. En el 26,1\% (12) de los afrocolombianos, el 22,2\% (5) de los indígenas y el 20,4\% (20) de los mestizos tienen astigmatismo. Del total de la población el 2,3\% (8) tienen astigmatismo en valores por fuera de lo fisiológico (astigmatismo mayor a $2.00 \mathrm{D}$ ).

La anisometropía se encontró en el 7,7\% del total de la población; representada por un $6,3 \%$ para el grupo mestizo (15), 7,1\% para el afrocolombiano
(7) y $11,8 \%$ para el indígena (2). La presencia de anisometropía esférica fue del $1,4 \%$ en el total de la población (5).

El porcentaje de prescripción óptica fue del 5,1\% (18) y el farmacológico $2,6 \%$ (9), representado principalmente por antialérgicos y paliativo del 3,2\% (11). El $3,4 \%$ registrado en remisiones fue para valoración y tratamiento por terapia visual. Al 14,5\% le recomendaron control por optometría en un plazo menor a un año.

En el estado de la motilidad ocular se encontró que la exoforia fisiológica está reportada en el $42 \%$ (148) de las historias, seguido por la ortoforia en el 36,9\% (130) y las forias tipo exoforia fuera de los valores fisiológicos en el 16,7\% (59). En total el $80 \%$ presentó un estado motor normal. 
Tabla 3. Frecuencia del astigmatismo por grupos étnicos

\begin{tabular}{ccccccc}
\hline & \multicolumn{2}{c}{ Afrocolombiano } & \multicolumn{2}{c}{ Indígena } & \multicolumn{2}{c}{ Mestizo } \\
\hline Cilindro & Frecuencia & Porcentaje & Frecuencia & Porcentaje & Frecuencia & Porcentaje \\
\hline$-0,25$ & 18 & 39 & 3 & 33,4 & 39 & 39,8 \\
$-0,5$ & 16 & 34,7 & 1 & 11,1 & 39 & 39,8 \\
$-0,75$ & 3 & 6,5 & 2 & 22,2 & 6 & 6,2 \\
-1 & 2 & 4,4 & 1 & 11,1 & 5 & 5,2 \\
$-1,25$ & 2 & 4,4 & 0 & 0,0 & 2 & 2,0 \\
$-1,5$ & 0 & 0,0 & 1 & 11,1 & 0 & 0,0 \\
$-1,75$ & 2 & 4,4 & 1 & 11,1 & 1 & 1,0 \\
-2 & 0 & 0,0 & 0 & 0,0 & 1 & 1,0 \\
$-2,25$ & 1 & 2,2 & 0 & 0,0 & 0 & 0,0 \\
$-2,5$ & 1 & 2,2 & 0 & 0,0 & 1 & 1,0 \\
-3 & 1 & 2,2 & 0 & 0,0 & 2 & 2,0 \\
$-3,25$ & 0 & 0,0 & 0 & 0,0 & 1 & 1,0 \\
\hline$-3,5$ & 0 & 0,0 & 0 & 0,0 & 1 & 1,0 \\
\hline Total por grupo & 46 & $100 \%$ & 9 & $100 \%$ & 98 & $100 \%$ \\
\hline
\end{tabular}

El estrabismo fue reportado en el 1,7\% de los casos en igual proporción, tanto para la endotropia como para la exotropia. Las endotropias fueron de tipo acomodativo refractivo. En cuatro casos no está determinado el estado de alineamiento ocular por falta de colaboración del paciente (Tabla 4).

Tabla 4. Frecuencia del estado motor

\begin{tabular}{lll}
\hline Estado Motor & Frecuencia & Porcentaje \\
\hline Condiciones motoras fisiológicas & 281 & 79,8 \\
Alteraciones motoras estrábicas & 6 & 1,7 \\
Alteraciones motoras no estrábicas & 61 & 17,3 \\
No colaboró & 4 & 1,0 \\
Total & 352 & 100,0 \\
\hline
\end{tabular}

Dentro de los grupos de edades la ortoforia fue el estado motor más frecuente a menor edad, presentándose en el $66,7 \%, 88,9 \%, 80 \%$ y $63,9 \%$ de los casos en los grupos de 12-18, 19-24, 25-30 y 31-36 meses, respectivamente, y disminuye hasta un $20 \%$ en el grupo de 61-72 meses. En el total de la población el 42,04\% presentó exoforia fisiológica y el 36,9\% fue ortofórico.

Se recopiló información de valoración de examen externo y fondo de ojo de 704 ojos, donde se halla- ron $21(3,0 \%)$ con blefaritis, $6(0,9 \%)$ con conjuntivitis bacteriana, $4(0,6 \%)$ con conjuntivitis alérgica en las etnias mestiza y afrocolombiana, 3 con ptosis palpebral (un caso bilateral y uno unilateral) y $1(0,1 \%)$ con conducto lagrimal obstruido. De segmento posterior no se hallaron reportes de alteraciones, ni diagnósticos de patologías en las historias clínicas.

\section{DISCUSIÓN}

El estado refractivo promedio de la población en este estudio (analizado vectorialmente) es la hipermetropía, coincidiendo con lo reportado por Morgan et al. ${ }^{6}$ y con Figueroa y Molina ${ }^{7}$ en el grupo de los 5 años de edad. Mientras en ambos estudios, con un porcentaje superior (80\%) al observado en esta investigación; el diagnóstico principal del estado de la refracción de la muestra corresponde a la emetropía con un $67,05 \%$. Lo anterior posiblemente se debe a que la mayor parte de los valores refractivos cayeron dentro del rango de poderes dióptricos considerados como emetropía.

Kleinstein et al. ${ }^{10}$ reportaron que el $36,9 \%$ de los hispanos (mestizos) presenta astigmatismo, más de cuatro veces a lo observado en este estudio $(8,4 \%)$, mientras que la prevalencia reportada por esos autores es de $12,7 \%$ para la hipermetropía y de $4,4 \%$ para 
la miopía, estos valores estuvieron más cercanos a los resultados del presente estudio en el que se halló 19,8\% y $2,5 \%$, respectivamente. Las discrepancias pueden deberse a algunas condiciones que difieren entre ambos estudios, tales como: la edad de la población fue mayor (de los 5 a los 17 años); en el caso de la miopía el porcentaje está posiblemente relacionado con el grupo de edad, donde, como lo han reportado en otros estudios, este estado tiende a ser más frecuente ${ }^{29}$. Para el caso de la hipermetropía, los autores mencionados la definieron a partir de valores superiores a los de este estudio, adicionalmente, como una diferencia procedimental, la refracción realizada por ellos se hizo bajo cicloplejia. Pese a que el criterio para el astigmatismo también fue alto, encontraron una prevalencia de más de 4 veces a la hallada en el presente estudio.

En otros trabajos, como el estudio MEPEDS ${ }^{29,30}$, donde la población de hispanos fue amplia (3030 sujetos de procedencia hispana), encontraron una prevalencia de la hipermetropía de $20,8 \%$, este valor es un poco más cercano a lo hallado en este trabajo, aunque sus valores diagnósticos de referencia para los tres estados refractivos fueron mayores en 1,00 D en la hipermetropía, 0,50 D miopía y 1,00 D astigmatismo, y también a que el examen refractivo lo determinaron con cicloplejia ${ }^{9}$. La prevalencia del astigmatismo de 12,7\% y de la miopía de 6,6\% fue superior a la de este estudio.

En una investigación realizada en Argentina por Verrone y Simi ${ }^{41}$, la prevalencia en niños de 6 años de la hipermetropía y astigmatismo fue de casi el 36\%, y $7,1 \%$ la miopía, todas superiores a lo aquí observado; sin embargo, con una muestra solamente de sujetos con agudeza visual disminuida. Por el contrario, en el de Antioquia ${ }^{26}$, donde la población fue de casos hallados positivos al tamizaje, presentaron una prevalencia para la hipermetropía del 9,2\% en el grupo de 0 a 5 años, muy inferior a la aquí reportada.

En el estudio realizado por Kleinstein et al. ${ }^{10}$ mencionado anteriormente, los autores reportaron en pacientes afroamericanos una prevalencia para la hipermetropía, astigmatismo y miopía del 6,4\%, 20\% y 6,6\%, respectivamente, en este estudio la prevalencia en el grupo étnico afrocolombiano para la hipermetropía fue superior con un $28,57 \%$ e inferior para el astigmatismo representado por el $12,24 \%$ y la miopía por el $4,08 \%$. El comportamiento fue similar al mestizo, excepto por una mayor prevalencia de la hipermetropía. En el estudio de MEPEDS ${ }^{29,30}$ reportaron una prevalencia de hipermetropía del 20,8\%, para el astigmatismo $12,7 \%$ y para la miopía $6,6 \%$, datos próximos a los del presente trabajo, como se observó al comparar el mismo estudio con los mestizos.

En un porcentaje mayor que en los afrocolombianos y los mestizos se observó la hipermetropía en los indígenas $(47,1 \%)$. Se encontraron pocos referentes bibliográficos de estudios en población indígena colombiana para comparar. Medina, en su estudio publicado en la página del Banco de la República realizado en las diferentes comunidades indígenas colombianas, reportó: "la mayoría de los examinados presentaron defectos refractivos bajos, siendo el principal la hipermetropía, generalmente menor $\mathrm{a}+1.50 \mathrm{D}$, la cual pudo asociarse a astigmatismos bajos contra la regla" ${ }^{\prime 1}$, se desconoce el año de realización o publicación del documento, así como el tamaño de la población evaluada y los grupos de edades. En este estudio, estado refractivo se encontró entre $0 \mathrm{y}+1,25 \mathrm{D}$; sin embargo, la muestra de la que se obtuvo la información es reducida y perteneció solo a el grupo indígena Embera Chamí, por lo cual, los resultados aquí reportados pueden no ser representativos de las poblaciones indígenas colombianas.

La prevalencia de la anisometropía de 7,7\% fue coincidente con la hallada por Abrahamsson, Fabian y Sjöstrand $^{42}$ e Ingram et $\mathrm{al}^{43}$, quienes en sus investigaciones hallaron aproximadamente del $7-11 \%$ de los niños de 1-4 años con anisometropía en la esfera de $1.00 \mathrm{D}$ o más. Aplicando el mismo criterio, el estudio MEPEDS ${ }^{44}$ reportó 4,3\% de anisometropía para los hispanos y 4,2\% para afroamericanos y Lasso ${ }^{26}$ del 4,4\%; todos estos datos inferiores a los hallados aquí; donde para el grupo mestizo fue de $6,3 \%, 7,1 \%$ para el afrodescendiente y $11,8 \%$ para el indígena, no se encontraron investigaciones relativas a la anisometropía en población indígena colombiana.

Huynh et al..$^{45}$ reportaron la anisometropía en un porcentaje muy inferior al aquí encontrado, para estos autores, esta se halla representada por el 1,6\%; sin embargo, la población estudiada perteneció a individuos blancos, europeos y asiáticos.

Las patologías más diagnosticadas en la población fueron la blefaritis con el 3,0\%, conjuntivitis bacteriana con $0,9 \%$ y conjuntivitis alérgica, que solo contó con el 
0,6\% en las etnias mestiza y afrocolombiana. Para Márque $^{46}$ la conjuntivitis alérgica se presentó en el 2,5\% de los registros médicos en el grupo de 1-4 años. La diferencia se puede explicar posiblemente, porque los datos en este último provienen de una institución a donde acuden los pacientes por alteraciones visuales y oculares.

En su estudio realizado en el litoral del Chocó, Buchelli y Corva en el $2009^{27}$, reportaron la presencia de la conjuntivitis bacteriana en aproximadamente $200 \mathrm{ca}-$ sos por cada 1000 individuos en la población indígena Wounana y la alérgica en 20 de cada 1000 del género femenino de la población afrocolombiana; mientras en este estudio tanto la blefaritis como las conjuntivitis bacteriana y alérgica solo involucraron 4 sujetos en las etnias mestiza y afrocolombiana.

La razón por la cual se registraron pocas patologías infecciosas y en su lugar, más alérgicas y de alteración del borde palpebral, comparado con Buchelli, se podría deber a las diferencias ambientales de las regiones en las cuales se ubicaban las poblaciones de los dos estudios. De igual modo, el estudio de Antioquia de 1998 ${ }^{26}$, presenta una prevalencia de la patología superior en el rango de 0 a 5 años con un 14,3\%, donde mencionan entre otras patologías la conjuntivitis alérgica, la catarata y la coriorretinitis; dicha prevalencia pudo deberse a que la población de estudio había sido clasificada como positiva para realizar examen optométrico.

En otras alteraciones del segmento anterior se observaron 3 ojos con ptosis palpebral con el $0,42 \%$ (un caso bilateral y uno unilateral) y $0,14 \%$ con conducto lagrimal obstruido. Solo algunos estudios identificaron la prevalencia de patologías oculares en Colombia por regiones, Lasso ${ }^{26}$, en Santo Domingo (Antioquia), reporta un $0,83 \%$ en los menores de 12 años detectados como positivos en el tamizaje visual. Otros estudios han identificado principalmente las patologías asociadas con ceguera y discapacidad visual ${ }^{19,31,1}$.

Con respecto al estado oculomotor, $\mathrm{MEPEDS}^{47} \mathrm{ob}$ servó un porcentaje de prevalencia del estrabismo en hispanos de 2,4\% y 2,5\% en individuos afroamericanos, análogo al 2,7\% que Lasso había reportado ${ }^{26}$. En este estudio el estrabismo estuvo presente en un porcentaje inferior de 1,7\%; adicionalmente, se encontraron en un alto porcentaje la exoforia fisiológica y la ortoforia, representadas cada una con $42,0 \%$ y $36,9 \%$, lo que denota un equilibrio oculomotor y desarrollo binocular dentro de parámetros normales. Esta información no se reporta comúnmente en las investigaciones.

La comparación de los resultados de este trabajo para los grupos de edades de los 12 a los 30 meses no se realizó con otras investigaciones debido al reducido número de registros en cada grupo.

El estado refractivo que más se presentó en todos los grupos de edad fue la emetropía, seguida por la hipermetropía. Los estudios refractivos realizados en otras poblaciones hispanas y afrodescendientes muestran mayores prevalencias para el astigmatismo y en menor frecuencia para la miopía, lo cual lleva a inferir que esos estudios no necesariamente representan la tendencia de las poblaciones mestiza y afrodescendiente colombiana en cuanto a sus características refractivas.

Las patologías presentes en segmento anterior más prevalentes corresponden a la blefaritis y las conjuntivitis y más frecuentes en las etnias mestiza y afrocolombiana.

Las patologías oculares, especialmente las de segmento posterior en mestizos y afrodescendientes muestran porcentajes de prevalencia menores a los reportados en los estudios de otros países, y como se manifestó en los defectos refractivos, de nuevo, puede llevar a inferir que esos estudios no necesariamente describen el comportamiento de nuestra población. En los estados oculomotores, la exoforia y, dentro de ella la fisiológica, fue la condición más diagnosticada.

\section{Financiación}

Apoyo financiero del Departamento de Proyección Social de la Fundación Universitaria del Área Andina Seccional Pereira.

\section{REFERENCIAS}

1. Zuluaga C, Sierra MV, Asprilla E. Causas de ceguera infantil en Cali, Colombia. Colomb Med. 2005;36(4):235-8.

2. Holden BA, Fricke TR, Ho SM, Wong R, Schlenther G, Cronjé $S$, et al. Global vision impairment due to uncorrected presbyopia. Arch Ophthalmol. 2008;126(12):1731-9. doi: 10.1001/archopht.126.12.1731. 
3. Ministerio de Salud. Resolución Número 4288 de 1996 [Internet]. Vol. 1996. 1996 [citado 2015 nov 25]. p. 1-8. Recuperado a partir de: https://www.minsalud.gov.co/ Normatividad_Nuevo/RESOLUCIÓN 4288 DE 1996.pdf

4. Gaviria A, Muñoz N, Ruiz F, Ospina M, Urquijo L, Arias J, et al. Plan Decenal de Salud Pública 2012-2021. Minist salud [Internet]. 2013;(32). Recuperado a partir de: http:// www.minsalud.gov.co/Documentos y Publicaciones/Plan Decenal - Documento en consulta para aprobación.pdf

5. $\quad$ OMS | Ceguera y discapacidad visual [Internet]. World Health Organization, 2014 [citado 2015 jul 28]. p. Nota descriptiva No 282. Recuperado a partir de: http://www. who.int/mediacentre/factsheets/fs282/es/

6. Morgan IG, RoseKA, Ellwein LB. Is emmetropia the natural endpoint for human refractive development? An analysis of population-based data from the refractive error study in children (RESC). Acta Ophthalmol. 2010;88(8):87784. doi: 10.1111/j.1755-3768.2009.01800.x.

7. Figueroa LF, Molina NP. Errores refractivos en niños de tres a siete años en la localidad de Chapinero de la ciudad de Bogotá. Cienc Tecnol para la Salud Vis y Ocul. 2011;9(2):55-61. doi: 10.19052/sv.174.

8. Multi-Ethnic Pediatric Eye Disease Study Group. Prevalence of Myopia and Hyperopia in 6- to 72-Month-Old African American and Hispanic Children: The Multi-Ethnic Pediatric Eye Disease Study. Ophthalmology. 2010;117(1):140-147.e3. doi: 10.1016/j. ophtha.2009.06.009.

9. Fozailoff A, Tarczy-Hornoch K, Cotter S, Wen G, Lin J, Borchert M, et al. Prevalence of Astigmatism in 6 to 72 Months Old African American and Hispanic Children: The Multi-Ethnic Pediatric Eye Disease Study. Ophthalmology. 2011;118(2):284-93. doi: 10.1016/j. ophtha.2010.06.038.

10. Kleinstein RN, Jones LA, Hullett S, Kwon S, Lee RJ, Friedman NE, et al. Refractive error and ethnicity in children. Arch Ophthalmol. 2003;121(8):1141-7.

11. Medina DM. Defectos refractivos y enfermedades oculares de las comunidades indígenas y negras de Colombia. [Citado 2015 jul 28]. Recuperado a partir de: http:// www.banrepcultural.org/blaavirtual/geografia/geofraf1/ defectos.htm

12. Razavi H, Kuper H, Rezvan F, Amelie K, Mahboobi-Pur $\mathrm{H}$, Oladi MR, et al. Prevalence and causes of severe visual impairment and blindness among children in the lorestan province of iran, using the key informant method. Ophthalmic Epidemiol. 2010;17(2):95-102. doi: $10.3109 / 09286581003624954$.
13. Dorairaj SK, Bandrakalli P, Shetty C, R V, Misquith D, Ritch R. Childhood blindness in a rural population of southern India: prevalence and etiology. Ophthalmic Epidemiol. 2008;15(3):176-82. doi: 10.1080/09286580801977668.

14. Rabiu M. Prevalence of blindness and low vision in North Central, Nigeria. West Afr J Med. 2008;27(4):238-44.

15. Lafuente F. Detección precoz de trastornos de la agudeza visual en escolares y su relación con el rendimiento escolar 3 ER año del EGB1 de la Qocha. Rev la Fac Med. 2007;8(1):5-9.

16. Dane. Censo general 2005. Discapacidad. Personas con limitaciones permanentes [Internet]. 2006 [citado 2015 jul 28]. p. 34. Recuperado a partir de: http://www.dane. gov.co/files/censo2005/discapacidad.pdf

17. Dane. Encuesta Nacional de Vida. 2010.

18. Williams C, Harrad RA, Harvey I, Sparrow JM. Screening for amblyopia in preschool children : Results of a population-pased, randomised controlled trial and the ALSPAC Study Team. Ophthalmic Epidemiol. 2001;8(5):279-96.

19. Tarczy-Hornoch K, Varma R, Cotter SA, McKean-Cowdin R, Lin JH, Borchert MS et al. Risk factors for decreased visual acuity in preschool children: The multi-ethnic pediatric eye disease and baltimore pediatric eye disease studies. Ophthalmology. 2011;118(11):2262-73. doi: 10.1016/j.ophtha.2011.06.033.

20. Rutstein RP. Contemporary issues in amblyopia treatment. Optometry. 2005;76(10):570-8. doi: 10.1016/j. optm.2005.08.017.

21. Salazar V, Zuazo AR. Detección de ambliopía en niños de 3 a 6 años en el "Hospital del Niño Dr. Ovidio Aliaga Uría”. Rev Soc Bol Ped. 2006;45(3):148-52.

22. Garvey KA., Dobson V, Messer DH, Miller JM, Harvey EM. Prevalence of strabismus among preschool, kindergarten, and first-grade Tohono O'odham children. Optometry. 2010;81(4):194-9. doi: 10.1016/j. optm.2009.10.010.

23. Kvarnstro G, Jakobsson P, Lennerstrand G. Visual screening of Swedish children: An ophthalmological evaluation. Acta Ophthalmol Scand. 2001;79:240-4.

24. Cotter SA., Varma R, Tarczy-Hornoch K, McKean-Cowdin R, Lin J, Wen G et al. Risk factors associated with childhood strabismus: The multi-ethnic pediatric eye disease and baltimore pediatric eye disease studies. Ophthalmology. 2011;118(11):2251-61. doi: 10.1016/j. ophtha.2011.06.032. 
25. Yekta A, Fotouhi A, Hashemi H, Dehghani C, Ostadimoghaddam $\mathrm{H}$, Heravian $\mathrm{J}$, et al. Prevalence of refractive errors among schoolchildren in Shiraz, Iran. Clin Exp Ophthalmol. 2010;38(3):242-8. doi: 10.1111/j.1442-9071.2010.02247.x.

26. Lasso E. Prevalencia de problemas visuales en menores de 12 años Santo Domingo Antioquia. CES Med. 1998;12(1):26-33.

27. Buchelli A, Corva D. Comparación de la prevalencia de patologías de segmento anterior y defectos refractivos en los indígenas Wounana y la población afrocolombiana del litoral del rio San Juan departamento del Chocó [Trabajo de Grado].Colombia: Universidad de La Salle; 2009.

28. Atkinson J, Anker S, Bobier W, Braddick O, Durden K, Nardini $M$, et al. Normal emetropization in infants with expectacle correction for hyperopia. Invest Opthtalmol Vis Sci. 2000;41(12):3726-31.

29. Leat SJ. To prescribe or not to prescribe? Guidelines for spectacle prescribing in infants and children. Clin Exp Optom. 2011;94(6):514-27. doi: 10.1111/j.1444-0938.2011.00600.x.

30. Mosquera JD. La población afrocolombiana. Boletín del Movimiento Nacional Afrocolombiano Cimarrón. Bogotá. [Citado 2015 ago 4]. Recuperado a partir de: http:// www.banrepcultural.org/blaavirtual/sociologia/estudiosafro/estudiosafro3.htm

31. Mosquera JD. El ser afrocolombiano. Boletín del Movimiento Nacional Afrocolombiano Cimarrón. Bogotá. [Citado 2015 ago 4]. Recuperado a partir de: http://www. banrepcultural.org/blaavirtual/sociologia/estudiosafro/ estudiosafro10.htm

32. Ulloa EA. Grupo indígena los Embera [Internet]. [Citado 2015 ago 4]. Recuperado a partir de: http://www.banrepcultural.org/blaavirtual/geografia/geograf/embera1.htm

33. Márquez-Galvis MM, Cáceres Díaz MC. Perfil de la salud visual y ocular de los niños de dos centros de desarrollo infantil de Pereira, Colombia. Cienc Tecnol para la Salud Vis y Ocul. 2017;15(2):61-70. doi: doi.org/10.19052/sv.4162.

34. Modalidades de la educación inicial - Primera infancia [Internet]. [Citado 2017 jul 10]. Recuperado a partir de: http://www.mineducacion.gov.co/primerainfancia/1739/ w3-article-228881.html

35. Primera infancia de grupos étnicos, en la agenda del Ministerio de Educación - Centro Virtual de Noticias de Educación [Internet]. [Citado 2017 jul 10]. Recuperado a partir de: http://www.mineducacion.gov.co/cvn/1665/ w3-article-300121.html
36. Hernández-Sampieri R, Fernández Collado C, Baptista Lucio P. Metodología de la investigación. 6th ed. Mc Graw Hill; 2014. 1-589 p.

37. ¿Qué es el Sisbén? [Internet]. [citadp 2017 jul 7]. Recuperado a partir de: https://www.sisben.gov.co/sisben/Paginas/Que-es.aspx

38. Páginas - Régimen contributivo [Internet]. [Citado 2017 jul 10]. Recuperado a partir de: https://www.minsalud.gov. $\mathrm{co} /$ proteccionsocial/Paginas/RégimenContributivo.aspx

39. Páginas - Aseguramiento al sistema general de salud [Internet]. [citadp 2015 jul 10]. Recuperado a partir de: https://www.minsalud.gov.co/proteccionsocial/Regimensubsidiado/Paginas/aseguramiento-al-sistema-general-salud.aspx.

40. Ministerio de Salud. Resolución 8430 de 1993 - 1.1993 p. 1-12.

41. Verrone P, Simi M. Prevalencia de agudeza visual baja y trastornos oftalmológicos en niños de seis años de la ciudad de Santa Fe. Arch Argent Pediatr. 2008;106(4):328-33.

42. Abrahamsson M, Fabian G, Sjöstrand J. A longitudinal study of a population based sample of astigmatic children. II. The changeability of anisometropia. Acta Ophthalmol. 1990;68(4):435-40.

43. Ingram RM, Traynar MJ, Walker C, Wilson JM. Screening for refractive errors at age 1 year: a pilot study. Br J Ophthalmol. 1979;63(4):243-50.

44. Borchert M, Tarczy-Hornoch K, Cotter SA, Liu N, Azen SP, Varma R; MEPEDS Group. Anisometropia in Hispanic and african american infants and young children: the multi-ethnic pediatric eye disease study. Ophthalmology. 2010 Jan;117(1):148-153.e1. doi: 10.1016/j. ophtha.2009.06.008.

45. Huynh SC, Wang XY, Ip J, Robaei D, Kifley A, Rose K a, et al. Prevalence and associations of anisometropia and aniso-astigmatism in a population based sample of 6 year old children. Br J Ophthalmol. 2006;90(5):597-601. doi: 10.1136/bjo.2005.083154.

46. Márquez M. Prevalencia de las alergias en la consulta clínica de optometría Universidad Santo Tomás Bucaramanga, 2001. Investig Andin. 2004;(9):22-6.

47. The Multi-Ethnic Pediatric Eye Disease Study Group - MEPEDS. Prevalence of Amblyopia and Strabismus in African American and Hispanic Children Ages 6 to 72 Months. Ophthalmology. 2008;115(7):1229-36. doi: 10.1016/j.ophtha.2007.08.001. 